\title{
Formulation, Development and Characterization of Ethosomes of Atorvastatin
}

\author{
Shivendra Agarwal ${ }^{1 *}$, Girendra Gautam ${ }^{1,2}$ \\ 'Department of Pharmacy, Bhagwant University Ajmer, Rajasthan, INDIA \\ ${ }^{2}$ Shri Ram College of Pharmacy, Department of Pharmacy, Muzaffarnagar, Uttar Pradesh, INDIA.
}

\begin{abstract}
Objectives: The main objective of the present investigation was to develop transdermal delivery of Atorvastatin, a HMG-CoAreductase inhibitor used in the treatment of congestive heart failure (CHF). Atorvastatin was formulated into ethosomal formulation to reduce the first pass metabolism and increase the systemic bioavailability. Methods: Cold method was used to formulate ethosomes by using suitable phosphatidylcholine and permeation enhancer. All the formulation were characterized for vesicle morphology, particle size and entrapment efficiency by Transmission Electron Microscopy, Differential light scattering and centrifugation respectively. The effect of different formulation variable on skin permeation of Atorvastatin was studied via synthetic semipermeable membrane or skin of new born mice by using diffusion cell. Results: The optimized ethosomal formulation showed enhanced drug delivery with no first pass metabolism, entrapment efficiency was found to be $98.65 \pm 0.63 \%$ and showed drug release
\end{abstract}

$98.13 \pm 3.67 \%$ for ethosomal formulation across rat skin as compared to $52.37 \pm 3.62 \%$ for hydroethanolic solution. Conclusion: Finally it was concluded from the study that, ethosomes can increase the transdermal flux, prolong the release and present an attractive route for sustained delivery of Atorvastatin.

Key words: Centrifugation, Scattering, Semipermiable, Transdermal, Bioavailability.

\section{Correspondence}

Mr. Shivendra Agarwal

Department of Pharmacy, Bhagwant University, Sikar Rd, Ajmer-305004, Rajasthan, INDIA.

Phone no: +91 8006033481

Email: agarwalshivacsr@gmail.com

DOI: 10.5530/ijpi.2020.2.29

\section{INTRODUCTION}

The technique of delivering a drug had a remarkable effect on its therapeutic efficacy. The therapeutic range of a drug is that at which it shows maximum therapeutic effect and dose above or below this concentration can be toxic or give no therapeutic effect at all. Due to retarded treatment of severe diseases, there is a growing demand of associative approach for the delivery of drug to targets in tissues. To decrease drug degradation, to enhance drug bioavailability and to arrest harmful side-effects there is a need to reinforce advanced drug targeting systems and various drug delivery for the faith of human life. Novel and Controlled Drug Delivery system are now an approach to provide a new life to the existing drug molecule. The various approaches for targeting the drug areniosomes, nanoparticles, ethosomes, lipososmes, microparticles, microcapsules and lipoproteins etc. ${ }^{1,2}$ As compared to oral drug delivery system, transdermal drug delivery system present favourable result as it abolish first pass metabolism and gastrointestinal disturbances of the therapeutic moiety. For TDDS stratum corneum is the main barrier which is required to be encountered as it allow passage to only lipophilic drugs having molecular weight $<500 \mathrm{Da}$. Advanced technique involving use of permeation enhancers are now popularly utilized to enhance the drug permeability across the skin easily. Now a days as compared to liposomes which are widely used to deliver drugs to the outer layers of skin, ethosomes and transferosomes are popularly employed to appreciate permeation across the stratum corneum barrier. ${ }^{1-3}$

\section{Ethosomes}

"Ethosomes are ethanolic liposomes". Ethosomes can be defined as noninvasive delivery carriers that enable drugs to reach deep into the skin layers and/or the systemic circulation. Ethosomes are utilized mainly for the enhanced delivery of medicinal agent across the skin membrane. In return to the need of advanced drug delivery system Touitou invented a vesicular system called ethosomes for topical drug delivery across the skin. Ethosomes are soft, smoothy, malleable vesicles utilized for novel delivery of medicaments.

Atorvastatin (Lipitor) is a member of the drug class known as statins, it is a HMG-CoAreductase inhibitor. It is used for lowering cholesterol. Atorvastatin is a competitive inhibitor of hydroxymethylglutarylcoenzyme A (HMG-CoA) reductase, the rate-determining enzyme in cholesterol biosynthesis via the mevalonate pathway. Atorvastatin acts primarily in the liver. The absolute bioavailability of atorvatsatin is approximately $14 \%$ and the systemic bioavailability of HMG-CoA reductase inhibitory activity is approximately $30 \%$. The low systemic bioavailability is due to presystemic clearance by gastrointestinal mucosa and first pass metabolism in the liver. Atorvastatin is most widely used in coronary heart disease (CHD).,5

\section{MATERIALS AND METHODS}

Atorvastatin was obtained as a gift sample from Chandra Labs, Hyderabad, India. Soya lecithin and other ingredients were obtained as a gift sample from Fine Chem Industries, Mumbai. Cholesterol was purchased from Cortex Laboratories, Hyderabad. All the other raw materials were of pharmacopoeia grade.

Pre-formulation study: Standardization of the drug was carried out using phosphate buffer $\mathrm{pH} 6.8$ by UV spectrophotometer (UV-160A, SHIMADZU). Solubility analysis of drug in various solvents including 
water, phosphate buffer $\mathrm{pH} 6.8$ and organic solvents like methanol was carried out.

\section{Formulation of Ethosomes \\ Screening of formulation component with Atorvastatin}

The foremost step was to screen different formulation component with Atorvastatin. In this step we screened different components with the drug Atorvastatin for the final selection of the excipients for the development of ethosomes of drug by cold method. The preliminary trial batches of ethosomes were prepared using selected process parameters to check the feasibility of preparation of ethosomes by using the available excipients that were also reported in earlier studies. ${ }^{6}$

\section{Drug-polymer interaction}

FTIR (Fourier Transform Infrared Spectroscopy): To determine the drug-polymer interaction IR septra of physical mixture of drug and polymers in combination were taken.

\section{Formulation of Ethosomes as drug carriers by cold method}

Atorvastatin loaded ethosomes were prepared by cold method followed by ultra-sonication. Phospholipids, drug and other lipid material were dissolved in ethanol in a covered beaker at a room temperature by vigorous stirring with the use of magnetic stirrer. Propylene glycol was added during stirring. This mixture was heated to $30^{\circ} \mathrm{C}$ in a water bath. The water heated to $30^{\circ} \mathrm{C}$ in a separate beaker was added to the mixture which was then stirred for $5 \mathrm{~min}$ in a covered vessel using magnetic stirrer. The vesicle size of ethosomal formulation was then reduced by ultrasonic probe sonicator for $10 \mathrm{~min}$ and off time was $10 \mathrm{sec}$. Finally the formulation was stored under refrigeration. The vesicles were observed under Carl Zeiss trinocular microscope at $40 \mathrm{X}$ and $100 \mathrm{X}$ magnification. ${ }^{7}$ Table 1 shows formula for formulation of Atorvastatin Ethsosomes.

\section{Characterization of optimized ethosomal drug delivery systems}

$p H$ : The $\mathrm{pH}$ of the prepared formulations after addition of all the ingredients was measured by using digital $\mathrm{pH}$ meter.

\section{Drug-Carrier Interaction Study}

Fourier Transformed Infrared Spectroscopy: Fourier Transformed Infrared Spectroscopy of ehosomes prepared sample and pure drug i.e Atorvastatin were attained using FTIR spectrophotometer.

Vesicle shape: Atorvastatin Ethosomal formulation shape was characterized and can be easily visualized by using transmission electron microscopy (TEM) or by differential scanning calorimetry (DSC).

\section{Elasticity measurement}

The elasticity of ethosomes vesicles were measured by extrusion method. The ethosomal formulation were extruded through filter membrane (pore size diameter- $100 \mathrm{~nm}$ ), using a stainless steel filter holder having
$50 \mathrm{~mm}$ diameter, by applying a pressure of $2.5 \mathrm{bar}$. The quantity of vesicle suspension, extruded in 5 min was measured.

Vesicle size and zeta potential: Vesicle size of the ethosomes can be determined by Malvern instrument. Zeta potential of the formulation can be measured by Malvern zeta sizer.

Drug entrapment: The entrapment efficiency of ethosomes can be measured by the ultracentrifugation technique. The entrapment efficiency (EE), of Atorvastatin ethosomes, was determined by measuring the concentration of free Atorvastatin in the dispersion medium. $1.0 \mathrm{ml}$ of the ethosomes was diluted up to $10 \mathrm{ml}$ with phosphate buffer $(\mathrm{pH}$ 5.5) and centrifuged at $14000 \mathrm{rpm}$ for $1 \mathrm{hr}$ to separate the lipid and aqueous phase. Supernatant was than filtered by $0.2 \mu$ membrane filter and analyzed by UV-VIS spectroscopy at $244 \mathrm{~nm}$.

$$
\% \mathrm{EE}=\frac{[\text { Initial drug }- \text { Free drug }]}{\text { Initial drug }} \times 100
$$

Where,

Initial drug $=$ mass of initial drug used for the assay.

Free drug $=$ mass of free drug detected in the supernatant after centrifugation of the aqueous dispersion.

\section{Drug content}

Drug content of the ethosomes can be determined using UV spectrophotometer. This can also be quantified by a modified high performance liquid chromatographic method. Ethosomal suspension (1 $\mathrm{ml}$ ) was taken and dissolved in $100 \mathrm{ml}$ methanol and shaken vigorously for $2 \mathrm{hr}$ in magnetic stirrer, there after the resultant solution was filtered and $1 \mathrm{ml}$ of sample was withdrawn and diluted to $10 \mathrm{ml}$ in a volumetric flask and the absorbance was recorded in UV spectrophotometer at 244 nm.

\section{In vitro release studies}

In-vitro drug release from the formulation was studied by the diffusion cell. Phosphate buffer $\mathrm{pH}$ (5.5) is used as diffusion medium, stirred at $50 \mathrm{rpm}$ at $37^{\circ} \mathrm{C} \pm 0.5^{\circ} \mathrm{C}$. The $5 \mathrm{ml}$ of ethosomal formulations were filled in test tubes and the end of the test tubes was covered with a dialysis membrane and was placed in such way that it touches the surface of diffusion medium present in receptor compartment. The drug sample were withdrawn at the interval of half an hour for the time period of 12 $\mathrm{hr}$ from diffusion medium and analyzed by using UV spectrophotometer at $244 \mathrm{~nm}$, using phosphate buffer $\mathrm{pH}(5.5)$ as a blank. ${ }^{8}$

\section{RESULTS}

\section{Drug-Carrier Interaction Study}

FTIR Study: It was found that there is no considerable change in the peak of the drug polymer mixtures when compared to pure Atorvastatin. FTIR spectra of the prepared drug polymer mixture shows that no

\begin{tabular}{|c|c|c|c|c|c|c|c|c|c|}
\hline \multirow[t]{2}{*}{ S.No. } & \multirow[t]{2}{*}{ Ingredient } & \multicolumn{5}{|c|}{ Formulation code } & \multirow[b]{2}{*}{ E6 } & \multirow[b]{2}{*}{ E7 } & \multirow[b]{2}{*}{ E8 } \\
\hline & & E1 & E2 & E3 & E4 & E5 & & & \\
\hline 1 & Atorvastatin (gm) & 20 & 20 & 20 & 20 & 20 & 20 & 20 & 20 \\
\hline 3 & Cholesterol (ml) & 15 & 30 & 45 & 15 & 30 & 45 & 15 & 45 \\
\hline 4 & Ethanol (ml) & 6 & 6 & 6 & 9 & 9 & 12 & 12 & 15 \\
\hline 6 & Distilled water & Upto $30 \mathrm{ml}$ & Upto $30 \mathrm{ml}$ & Upto $30 \mathrm{ml}$ & Upto $30 \mathrm{ml}$ & Upto $30 \mathrm{ml}$ & Upto $30 \mathrm{ml}$ & Upto $30 \mathrm{ml}$ & Upto $30 \mathrm{ml}$ \\
\hline
\end{tabular}

Table 1: Formulation of Atorvastatin Ethsosomes. 
change occurred in chemical structure. Thus it can be concluded that, according to FTIR studies there is no physico-chemical interaction between Atorvastatin and the polymers.

Evaluation of ethosomes: The optimized ethosomes were evaluated for particle size, particle shape, zeta potential and elasticity measurement. Table 2 shows the particle size of various formulation determined by scanning electron microscopy. Elasticity of ethosomal formulation was shown in Table 3, Figure 1 shows particle size determined by scanning eletron microscopy and zeta potential of atorvastain ethosomal formulation was shown in Figure 2.

Drug entrapment: The entrapment efficiency of different vesicular formulation was calculated as percent total drug entrapped. The data indicate that entrapment efficiency depends on ethanol concentration,

Table 2: Particle sizes of Atorvastatin ethosomal formulations.

\begin{tabular}{cc}
\hline Formulation Code & Particle size \\
\hline E1 & 94.64 \\
E2 & 112.52 \\
E3 & 153.63 \\
E4 & 149.75 \\
E5 & 95.6 \\
E6 & 117.85 \\
E7 & 94.62 \\
E8 & 104.69 \\
\hline
\end{tabular}

Table 3: Elasticity of Atorvastatin ethosomal formulations.

\begin{tabular}{cc}
\hline Formulation Code & Elasticity \\
\hline E1 & 84.72 \\
E2 & 80.93 \\
E3 & 86.07 \\
E4 & 86.4 \\
E5 & 89.9 \\
E6 & 84.68 \\
E7 & 84.33 \\
E8 & 83.91 \\
\hline
\end{tabular}

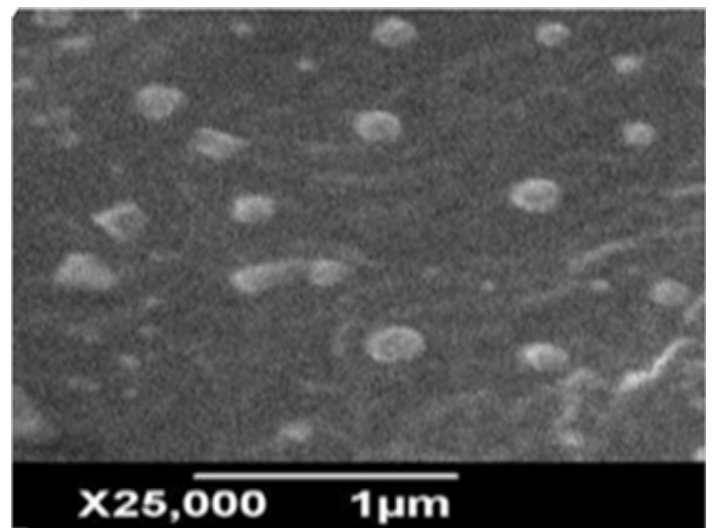

Figure 1: Scanning electron microscopy of Atorvastatin Ethosomal preparation. as the concentration increases up to $30 \%$, results increases in entrapment efficiency of ethosomal formulation. With further increase in ethanol concentration entrapment efficiency decreases, owing to increase fluidity of membrane and vesicles become more permeable that leads to decrease in entrapment efficiency of ethosomal formulation. Table 4 shows entrapment efficiency of ethosomal formulation.

In-vitro release studies: The in-vitro release study suggested that the penetration enhancing effect might be of greater importance to enhance skin delivery of atorvastatin by ethosomal vesicles under non occlusive condition, than intact vesicle permeation into the stratum corneum (SC). Figure 3 shows in-vitro drug release profile of atorvastatin ethosomal formulation no. 1,2,3,4 and in-vitro drug release profile of atorvastatin ethosomal formulation no. 5,6,7,8 was shown in Figure 4.

\section{DISCUSSION}

Atorvastatin having low bioavailability due to first pass metabolism was formulated into ethosomal gel to penetrate it through the skin, it was formulated into ethosomes using soya lecithin a phospholipid and ethanol as permeation enhancer. It was observed by various data obtained that ethosomes were of suitable size and shape to penetrate across the skin, the FTIR studies showed that there is no structural change in the drug after combining with various excipients. The zeta potential showed that ethsosomes were having suitable negative charge to remain as smaller vesicles and the drug release profile of atorvastatin as ethosomal formulation was found to be $98.13 \pm 3.67 \%$ as compared to conventional formulation $52.37 \pm 3.62 \%$.
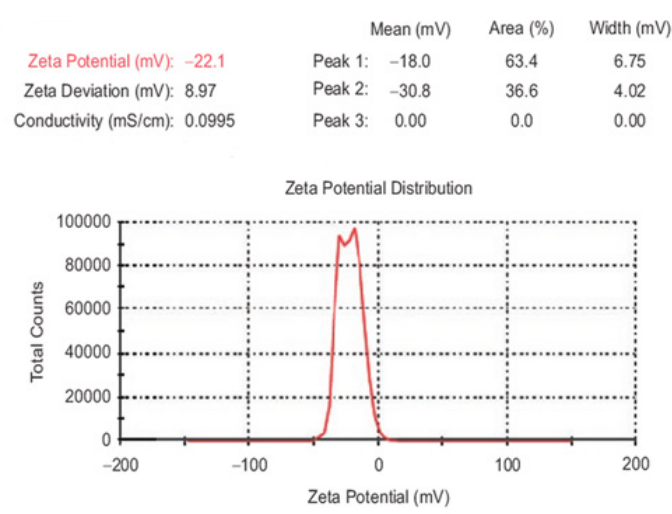

Figure 2: Zeta potential of prepared Atorvastatin ethosomes.

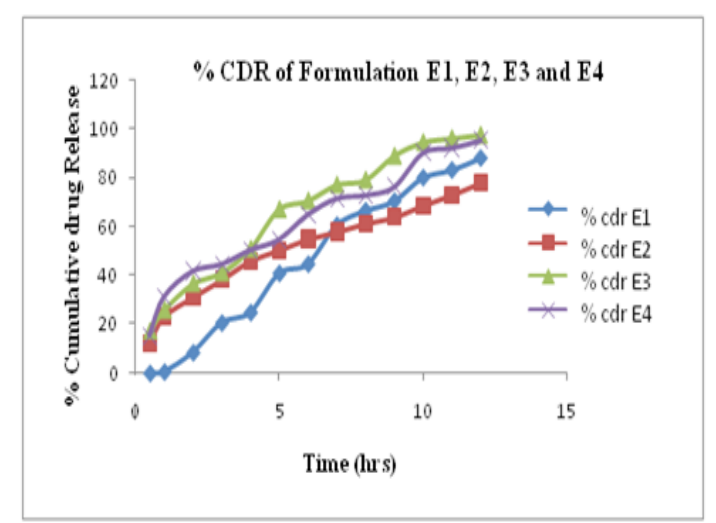

Figure 3: \%CDR of Atorvastatin ethosomal formulation E1, E2, E3 and E4. 


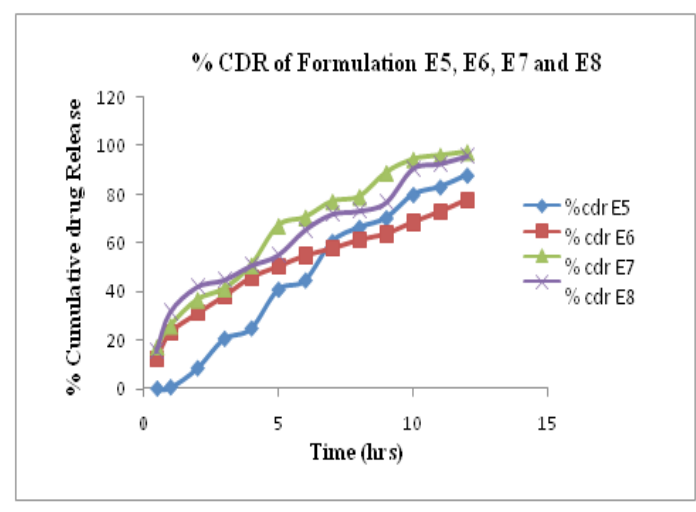

Figure 4: \%CDR of Atorvastatin ethosomal formulation E5, E6, E7 and E8.

Table 4: Entrapment efficiency of atorvastatin ethosomal formulations.

\begin{tabular}{cc|}
\hline Formulation & EE (\%) \\
\hline E1 & 97.8 \\
E2 & 96.44 \\
E3 & 98.58 \\
E4 & 97.48 \\
E5 & 98.65 \\
E6 & 96.45 \\
E7 & 98.04 \\
E8 & 96.65 \\
\hline
\end{tabular}

\section{CONCLUSION}

Based on the observation it was concluded that ethosomal formulation of atorvastatin is more effective than any other conventional formulation. The prepared formulation were characterized for various evolutionary parameters like vesicle size, entrapment efficiencies, vesicles elasticity, in-vitro drug deposition study and rate of transdermal flux across stratum corneum and prepared formulation were also characterized for in-vitro release studies by using cellophane membrane (semiperrmeable membrane), skin of new born mice for in-vitro study. From in-vitro drug release studies, it was concluded that by changing the ratio of Phospholipid and ethanol, atorvastatin release can be controlled for a prolonged period of time by reducing possible side effects occurred during conventional therapy.

\section{ACKNOWLEDGEMENT}

I would like to express deepest appreciation and sense of gratitude to my guide Prof. Girendra Kumar Gautam, I also pay sincere gratitude to all professors and experts for their efforts and contribution.

\section{CONFLICT OF INTEREST}

The authors declare none.

\section{ABBREVIATIONS}

FTIR: Fourier Transform Infrared Spectroscopy; TDDS: Transdermal drug delivery system; CDR: Cumulative drug release.

\section{REFERENCES}

1. Reddy PD, Swarnalatha D. Recent advances in Novel Drug Delivery Systems. J Physiother Phys Rehabil. 2010;2(3):2025-7.

2. Muller CC. Physicochemical characterization of colloidal drug delivery systems such as reverse micelles, vesicles, liquid crystals and nanoparticles for topical administration. Eur J Pharm Biopharm. 2004;58(2):343-56.

3. Asbill CS, Kattan AFE, Michniak B. Enhancement of transdermal drug delivery: Chemical and physical approaches. Critical Reviews in Therapeutic Drug Carrie Systems. 2000;17(6):621-58

4. Touitou E, Dayan N, Levi-Schaffer F, Piliponsky A. Novel lipid vesicular system for enhanced delivery. Journal of Lipid Research. 1998;8:113

5. Verma P, Pathak K. Therapeutic and cosmeceutical potential of ethosomes: An overview. J Lipid Res. 2010;1(3):274-82

6. Jain S, Umamaheshwari RB, Bhadra D, Jain NK. Ethosomes: A novel vesicular carrier for enhanced transdermal delivery of an anti-HIV agent. Indian J Pharm Sci. 2004;66(1):72-81.

7. Rastogi V, Yadav P. Transdermal drug delivery system: An overview. Asian Journal of Pharmaceutics. 2012;6(3):161-70.

8. Arunachalam A, Karthikeyan M, Kumar VD, Prathap M, Sethuraman S, et al. Transdermal Drug Delivery System: A Review. Cur Pharm Res. 2010;1(1):70-81. 